\title{
A Short Path to Erythrina Alkaloid Derivatives
}

\author{
Le Anh Tuan ${ }^{\dagger, *}$ and Guncheol Kim ${ }^{\dagger, *}$ \\ ${ }^{\dagger}$ Department of Chemistry, College of Natural Science, Chungnam National University, Daejon 305-764, Korea \\ *E-mail: guncheol@cnu.ac.kr \\ ¥Institute of Chemistry, Vietnamese Academy of Science and Technology (VAST), Hanoi, Vietnam \\ Received March 19, 2010, Accepted March 29, 2010
}

Key Words: Erythrina alkaloids, Arylation, Heck reaction, Erysotramidine, Iso-13-demethoxyerythratidinone

Erythrina alkaloids which display a variety of biological activity including hypnotic and CNS activity ${ }^{1}$ have drawn attraction for the synthesis over the years. Some of the recent strategies on the construction of the core spirocyclic structure include intramolecular cyclization reactions such as radical cyclizations, ${ }^{2}$ electrophilic substitution cyclizations on $N$-acyliminium intermediates or Pummerer-induced cyclizations, ${ }^{3}$ Heck reactions, ${ }^{4}$ and anionic substitution reactions. ${ }^{5}$

A few years ago, we developed a new route to the spirocyclic skeleton by palladium-catalyzed arylation of $\alpha, \beta$-unsaturated $\gamma$-lactam. ${ }^{6}$ In the route, the precursors for cyclization have been prepared by condensation of arylamine and keto-ester intermediates $^{3 \mathrm{c}}$ under reflux in toluene in the presence of $\mathrm{TsOH}$ (Scheme 1). ${ }^{3 \mathrm{~d}}$

For the synthetic application of this method toward erythrina alkaloids, we wanted to reinvestigate the palladium catalyzed cyclization of the requsite precursor which would be formed from the condensation of ketoester $\mathbf{1}$ and bromo-arylamine $\mathbf{2}$. In the reaction, two main products were formed as shown in the Scheme 2 , and the separated yields were $34 \%$ of compound 3

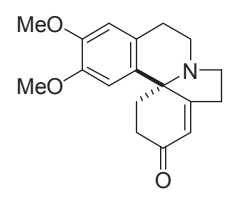

13-Demethoxyerythratidinone<smiles>CCC1Cc2cc(OC)c(OC)cc2[C@@]12CC=C[C@H](OC)C2</smiles>

Erysotramidine

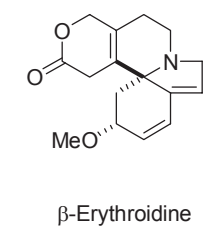

Figure 1

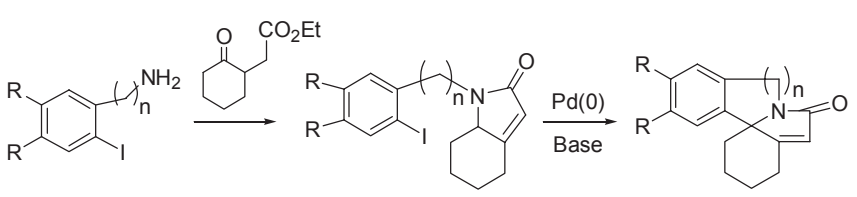

Scheme 1

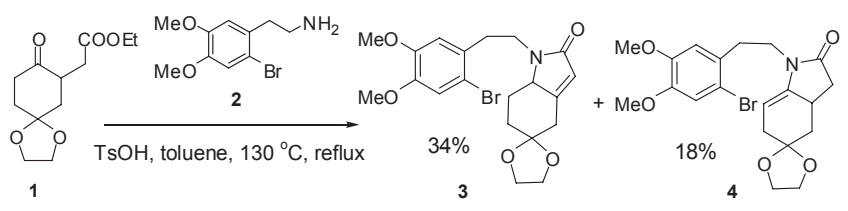

Scheme 2 and $18 \%$ of compound 4 . For the selective formation of each isomer, we have tried the cyclization reaction under different conditions by changing solvent, catalyst, and temperature. However, the ratio did not shift favorably for the purpose, so we considered that we had better find ways to transform each isomer to proper natural products or derivatives. It was envisioned that major intermediate 3 would be a proper precursor for erysotramidine and the minor intermediate 4 for iso-13-demethoxyerythratidinone (Scheme 2).

When the intermediate 3 was treated with $\mathrm{Pd}(\mathrm{OAc})_{2}$ in $\mathrm{DBU}$, enol intermediate was formed in 30\% yield through $\gamma$-lactam enolate formation followed by cyclization. Some amount of the corresponding ketal compound, the precursor of the enol intermediate, could be obtained if the reaction process was quenched earlier. However, the ketal interemediate was found to be reluctant to hydrolyse to ketone. Treatment of $\mathbf{5}$ with

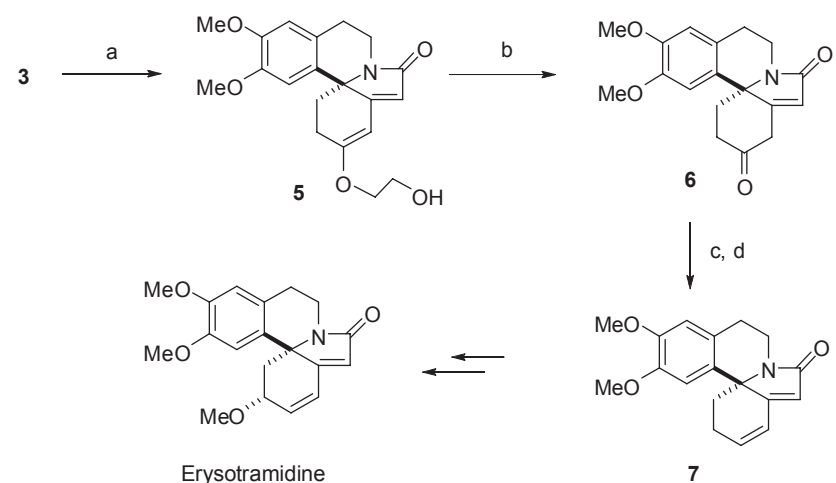

Scheme 3. Reagents and conditions (a) $\mathrm{Pd}(\mathrm{OAc})_{2}$, DBU, $140{ }^{\circ} \mathrm{C}, 15 \mathrm{~h}$, $30 \%$; (b) TsOH, acetone, reflux, $6 \mathrm{~h}, 71 \%$; (c) $\mathrm{NaBH}_{4}, \mathrm{CeCl}_{3} \cdot 7 \mathrm{H}_{2} \mathrm{O}$, $\mathrm{MeOH}, 3$ h, dr 2.6:1; (d) $\mathrm{POCl}_{3}, \mathrm{DBU}, \mathrm{CH}_{2} \mathrm{Cl}_{2}, 3$ h, 64\% (2 steps)

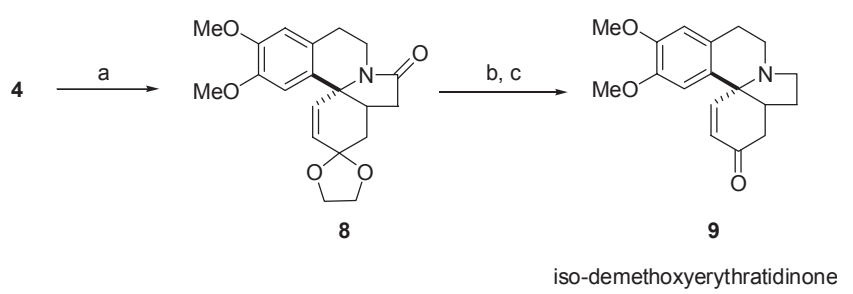

Scheme 4. Reagents and conditions (a) $\mathrm{Pd}(\mathrm{OAc})_{2}, \mathrm{PPh}_{3}, \mathrm{DBU}, 140{ }^{\circ} \mathrm{C}$, 15 h, 87\%; (b) $\mathrm{LiAlH}_{4}-\mathrm{AlCl}_{3}$, THF; (c) TsOH, acetone, $80{ }^{\circ} \mathrm{C}, 2 \mathrm{~h}$, $80 \%$ (2 steps) 
$\mathrm{TsOH}$ in acetone afforded compound $\mathbf{6}$ in $71 \%$ yield. Reduction of the carbonyl compound $\mathbf{6}$ under Luche condition, affording a mixture of diasteromers in 2.6:1 ratio, was followed by elimination to afford the known intermediate 7 for erysotramidine in $64 \%$ yield (Scheme 3$)^{7}$

Meanwhile, intermediate 4 was subjected to the conventional Heck reaction, yielding the 6-membered quaternary structure 8 in $87 \%$ yield rather than 7 -membered ring compound. Reduction of the amide group of $\mathbf{8}$ by $\mathrm{LAH} / \mathrm{AlCl}_{3}$ to amine was followed by deprotection of ketal under acid to iso-13-demethoxyerythratidinone ${ }^{8}$ in $80 \%$ in two steps.

In conclusion, we have prepared two intermediates from condensation of compound $\mathbf{1}$ and 2, and suggested concise routes to the synthesis of erysotramidine and iso-13-demethoxyerythratidinone through $\mathrm{Pd}$-mediated cyclization of the intermediates.

\section{Experimental Section}

1'-(2-Bromo-4,5-dimethoxyphenethyl)-1',6',7',7a'-tetrahydrospiro[[1,3] dioxolane-2,5'-indol $]-2^{\prime}\left(4^{\prime} H\right)$-one (3) and 1'(2-bromo-4,5-dimethoxyphenethyl)-3',3a',4',6'-tetrahydrospiro [[1,3] dioxolane-2,5'-indol]-2'(1'H)-one (4). To a solution of 1 $(2 \mathrm{~g}, 8.25 \mathrm{mmol})$ in anhydrous toluene $(30 \mathrm{~mL})$ were added TsOH (314 mg, $1.65 \mathrm{mmol}$ ) and 2-(2-bromo-4,5-dimethoxyphenyl)ethanamine 2 (2.36 g, $9.08 \mathrm{mmol})$. The reaction mixture was refluxed for $15 \mathrm{~h}$ using Dean-Stark trap. The solvent was evaporated under reduced pressure and the resultant crude material was purified by silica gel column chromatography with $n$-hexane/EtOAc (1:1 to 1:3), EtOAc $100 \%$ and EtOAc/MeOH (20:1) to give $3(1.23 \mathrm{~g}, 34 \%)$ and $4(651 \mathrm{mg}, 18 \%)$.

Compound 3: ${ }^{1} \mathrm{H}-\mathrm{NMR}\left(400 \mathrm{MHz}, \mathrm{CDCl}_{3}\right) \delta 6.99(1 \mathrm{H}, \mathrm{s})$, $6.76(1 \mathrm{H}, \mathrm{s}), 4.91(1 \mathrm{H}, \mathrm{m}), 4.06-3.92(4 \mathrm{H}, \mathrm{m}), 3.86(3 \mathrm{H}, \mathrm{s}$, OMe), 3.85 (3H, s, OMe), $3.76(1 \mathrm{H}, \mathrm{m}), 3.50(1 \mathrm{H}, \mathrm{m}), 3.15-2.82$ $(3 \mathrm{H}, \mathrm{m}), 2.58(1 \mathrm{H}, \mathrm{dd}, J=6.8,16.4 \mathrm{~Hz}), 2.50-2.32(2 \mathrm{H}, \mathrm{m})$, $2.17(1 \mathrm{H}, \mathrm{dd}, J=10.4,16.4 \mathrm{~Hz}), 2.06(1 \mathrm{H}, \mathrm{dd}, J=4.8,12.4$ $\mathrm{Hz}), 1.64(1 \mathrm{H}, \mathrm{t}, J=12,4 \mathrm{~Hz}) .{ }^{13} \mathrm{C}-\mathrm{NMR}\left(100 \mathrm{MHz}, \mathrm{CDCl}_{3}\right) \delta$ $174.1,148.3,148.1,141.1,129.6,115.2,113.9,113.2,107.8$, 94.1, 64.3, 64.1, 55.9, 55.8, 39.4, 36.5, 36.1, 34.6, 32.9, 32.6.

Compound 4: ${ }^{1} \mathrm{H}-\mathrm{NMR}\left(400 \mathrm{MHz}, \mathrm{CDCl}_{3}\right) \delta 7.00(1 \mathrm{H}, \mathrm{s})$, $6.81(1 \mathrm{H}, \mathrm{s}), 5.85(1 \mathrm{H}, \mathrm{s}), 4.05-3.89(4 \mathrm{H}, \mathrm{m}), 3.85(3 \mathrm{H}, \mathrm{s}$, $\mathrm{OMe}), 3.84(3 \mathrm{H}, \mathrm{s}, \mathrm{OMe}), 3.67(1 \mathrm{H}, \mathrm{dd}, J=6.0,12.0 \mathrm{~Hz}), 3.40$ $(1 \mathrm{H}, \mathrm{m}), 2.92(3 \mathrm{H}, \mathrm{m}), 2.81(1 \mathrm{H}, \mathrm{dd}, J=2.4,14.0 \mathrm{~Hz}), 2.53$ $(1 \mathrm{H}, \mathrm{dd}, J=2.0,14.0 \mathrm{~Hz}), 2.35(1 \mathrm{H}, \mathrm{m}), 1.85(1 \mathrm{H}, \mathrm{m}), 1.71$ $(1 \mathrm{H}, \mathrm{ddd}, J=3.6,14.0,17.6 \mathrm{~Hz}), 1.27(1 \mathrm{H}, \mathrm{m}) .{ }^{13} \mathrm{C}-\mathrm{NMR}(100$ $\left.\mathrm{MHz}, \mathrm{CDCl}_{3}\right) \delta 171.4,158.3,148.5,148.2,129.9,120.7,115.4$, $113.9,113.5,109.5,64.8,64.6,61.5,56.1,40.1,38.2,34.8,27.7$.

(S)-3-(2-Hydroxyethoxy)-1,2,8,9-tetrahy dro-11,12-dimethoxyindolo[1-a]isoquinolin-6-one (5). A mixture of 2 (548 $\mathrm{mg}, 1.25 \mathrm{mmol})$ and $\mathrm{Pd}(\mathrm{OAc})_{2}(14 \mathrm{mg}, 0.063 \mathrm{mmol})$ in $5 \mathrm{~mL}$ DBU in a sealed tube was heated at $140{ }^{\circ} \mathrm{C}$ for $15 \mathrm{~h}$. The reaction mixture was quenched with $40 \mathrm{~mL}$ aqueous $\mathrm{HCl} 2 \mathrm{M}$ solution, and then extracted with $\mathrm{CH}_{2} \mathrm{Cl}_{2}(30 \mathrm{~mL} \times 3)$. The organic layers were dried over anhydrous $\mathrm{MgSO}_{4}$ and concentrated in vacuo. The resulting residue was purified by silica gel column chromatography (EtOAc/MeOH 20:1 to 10:1) to afford 5 (132 mg, $30 \%)$ as a brownish liquid. ${ }^{1} \mathrm{H}-\mathrm{NMR}\left(400 \mathrm{MHz}, \mathrm{CDCl}_{3}\right) \delta 7.0$ $(1 \mathrm{H}, \mathrm{s}), 6.71(1 \mathrm{H}, \mathrm{s}), 5.96(1 \mathrm{H}, \mathrm{s}), 5.73(1 \mathrm{H}, \mathrm{s}), 4.15-3.91(5 \mathrm{H}$, m), $3.85(3 \mathrm{H}, \mathrm{s}, \mathrm{OMe}), 3.75(3 \mathrm{H}, \mathrm{s}, \mathrm{OMe}), 3.56(1 \mathrm{H}, \mathrm{m}), 2.98$
$(2 \mathrm{H}, \mathrm{m}), 2.41-2.25(2 \mathrm{H}, \mathrm{m}), 1.90(1 \mathrm{H}, \mathrm{m}), 1.79-1.61(1 \mathrm{H}, \mathrm{m})$. ${ }^{13} \mathrm{C}-\mathrm{NMR}\left(100 \mathrm{MHz}, \mathrm{CDCl}_{3}\right) \delta 172.2,163.6,159.4,148.1$, $146.6,128.7,126.5,115.0,112.0,108.4,95.4,69.5,64.5,60.7$, $55.9,55.8,37.3,33.4,27.0,26.9$.

(S)-1,2,8,9-Tetrahydro-11,12-dimethoxy-4H-indolo[1-a]isoquinoline-3,6-dione (6). A solution of 5 (132 $\mathrm{mg}, 0.37 \mathrm{mmol})$ and $\mathrm{TsOH}(10 \mathrm{mg})$ in acetone $(10 \mathrm{~mL})$ was refluxed at $60{ }^{\circ} \mathrm{C}$ for $6 \mathrm{~h}$. The reaction mixture was concentrated in vacuo and the resultant residue was purified by silica gel column chromatography with EtOAc 100\% to yield compound 6 (82 mg, 71\%) as a yellowish liquid. ${ }^{1} \mathrm{H}-\mathrm{NMR}\left(400 \mathrm{MHz}, \mathrm{CDCl}_{3}\right) \delta 6.70(1 \mathrm{H}$, s), $6.55(1 \mathrm{H}, \mathrm{s}), 6.25(1 \mathrm{H}, \mathrm{s}), 4.33(1 \mathrm{H}, \mathrm{ddd}, J=4.0,8.4,13.6$ $\mathrm{Hz}), 3.86$ (3H, s, OMe), 3.77 (3H, s, OMe), 3.42 (1H, dt, $J=8.0$, $13.6 \mathrm{~Hz}), 3.22(2 \mathrm{H}, \mathrm{s}), 3.11(1 \mathrm{H}, \mathrm{dt}, J=8.4,16.8 \mathrm{~Hz}),(1 \mathrm{H}, \mathrm{m})$, 2.90 (1H, ddd, $J=4.0,8.0,16.8 \mathrm{~Hz}), 2.58-2.52(2 \mathrm{H}, \mathrm{m}), 2.48$ $(1 \mathrm{H}, \mathrm{m}), 2.34(1 \mathrm{H}, \mathrm{m}) .{ }^{13} \mathrm{C}-\mathrm{NMR}\left(100 \mathrm{MHz}, \mathrm{CDCl}_{3}\right) \delta 197.4$, 170.2, 158.3, 149.1, 146.9, 126.3, 126.1, 125.3, 113.3, 108.0, 63.8, 56.1, 56.0, 37.0, 35.1, 34.9, 33.6, 25.6.

(S)-1,2,8,9-Tetrahydro-11,12-dimethoxyindolo[1-a]isoqui nolin-6-one (7). To a solution of $6(82 \mathrm{mg}, 0.26 \mathrm{mmol})$ in anhydrous $\mathrm{MeOH}$ was added $\mathrm{CeCl}_{3} .7 \mathrm{H}_{2} \mathrm{O}(108 \mathrm{mg}, 0.29 \mathrm{mmol})$. The mixture was stirred for $30 \mathrm{~min}$ before the addition of $\mathrm{NaBH}_{4}$ (11 mg, $0.29 \mathrm{mmol})$. After $3 \mathrm{~h}$, the reaction mixture was extracted with EtOAc $(30 \mathrm{~mL} \times 3)$, dried over anhydrous $\mathrm{Na}_{2} \mathrm{SO}_{4}$ and concentrated in vacuo. The crude product was dissolved in anhydrous $\mathrm{CH}_{2} \mathrm{Cl}_{2}(4 \mathrm{~mL})$ followed by addition of $\mathrm{POCl}_{3}(29 \mu \mathrm{L}$, $0.31 \mathrm{mmol})$ and DBU (191 $\mu \mathrm{L}, 1.3 \mathrm{mmol})$ consecutively. After $3 \mathrm{~h}$, the reaction mixture was evaporated under reduced pressure and purified by silica gel column chromatography (EtOAc/ $\mathrm{MeOH} 30: 1$ to $15: 1)$ to give 7 (51 mg, 64\%) as a colorless liquid. ${ }^{1} \mathrm{H}-\mathrm{NMR}\left(400 \mathrm{MHz}, \mathrm{CDCl}_{3}\right) \delta 7.01(1 \mathrm{H}, \mathrm{s}), 6.82(1 \mathrm{H}, \mathrm{dd}, J=$ 2.8, $10 \mathrm{~Hz}), 6.70(1 \mathrm{H}, \mathrm{s}), 6.30(1 \mathrm{H}, \mathrm{m}), 5.89(1 \mathrm{H}, \mathrm{s}), 4.04(1 \mathrm{H}$, $\mathrm{dt}, J=7.2,12.8 \mathrm{~Hz}), 3.86(3 \mathrm{H}, \mathrm{s}, \mathrm{OMe}), 3.77$ (3H, s, OMe), $3.56(1 \mathrm{H}, \mathrm{dt}, J=6.8,12.8 \mathrm{~Hz}), 2.99(2 \mathrm{H}, \mathrm{t}, J=6.8 \mathrm{~Hz}), 2.42$ $(1 \mathrm{H}, \mathrm{dt}, J=5.6,19.2 \mathrm{~Hz}), 2.33(1 \mathrm{H}, \mathrm{dd}, J=4.8,12.4 \mathrm{~Hz}), 2.21$ $(1 \mathrm{H}, \mathrm{m}), 1.85(1 \mathrm{H}, \mathrm{dt}, J=5.6,12.4 \mathrm{~Hz}) .{ }^{13} \mathrm{C}-\mathrm{NMR}(100 \mathrm{MHz}$, $\left.\mathrm{CDCl}_{3}\right) \delta 171.2,157.9,148.2,146.8,136.0,128.7,126.3,124.0$, 118.9, 112.1, 108.7, 64.6, 56.0, 55.9, 37.0, 35.0, 27.2, 24.6.

11',12'-Dimethoxy-4a',5',8',9'-tetrahydrospiro[[1,3]dioxolane-2,3'-indolo[1-a]isoquinolin]-6'(4'H)-one (8). A mixture of 4 (173 mg, $0.4 \mathrm{mmol}$ ), $\mathrm{PPh}_{3}(21 \mathrm{mg}, 0.08 \mathrm{mmol}), \mathrm{Pd}(\mathrm{OAc})_{2}$ $(9 \mathrm{mg}, 0.04 \mathrm{mmol})$ and DBU $(3 \mathrm{~mL})$ in a sealed tube was heated at $140{ }^{\circ} \mathrm{C}$ for $15 \mathrm{~h}$. The reaction mixture was treated with aqueous $\mathrm{HCl} 2 \mathrm{M}$ solution until $\mathrm{pH}=2$, then extracted with $\mathrm{CHCl}_{3}$ $(30 \mathrm{~mL} \times 3)$, dried over anhydrous $\mathrm{Na}_{2} \mathrm{SO} 4$ and concentrated in vacuo. The resulting residue was purified by silica gel column chromatography (EtOAc/MeOH 20:1 to 10:1) to afford 8 (124 $\mathrm{mg}, 87 \%)$ as a colorless liquid. ${ }^{1} \mathrm{H}-\mathrm{NMR}\left(400 \mathrm{MHz}, \mathrm{CDCl}_{3}\right) \delta$ $6.78(1 \mathrm{H}, \mathrm{s}), 6.59(1 \mathrm{H}, \mathrm{s}), 5.85(1 \mathrm{H}, \mathrm{d}, J=10.4 \mathrm{~Hz}), 5.77(1 \mathrm{H}$, $\mathrm{d}, J=10.4 \mathrm{~Hz}), 4.35(1 \mathrm{H}, \mathrm{m}), 4.12-3.97(4 \mathrm{H}, \mathrm{m}), 3.86(3 \mathrm{H}, \mathrm{s}$, $\mathrm{OMe}), 3.85$ (3H, s, OMe), 3.06-2.83 (3H, m), 2.68-2.60 (2H, m), 2.49-2.39 (2H, m), 2.09 (1H, dd, $J=4.8,14.8 \mathrm{~Hz}) .{ }^{13} \mathrm{C}-\mathrm{NMR}$ $\left(100 \mathrm{MHz}, \mathrm{CDCl}_{3}\right) \delta 172.9,148.2,147.9,131.0,130.3,126.7$, 126.2, 111.9, 109.3, 103.2, 64.9, 64.3, 61.1, 56.0, 55.9, 39.1, $36.1,35.1,34.1,28.6$.

Iso-demethoxyerythratidinone (9). To a solution of anhydrous $\mathrm{AlCl}_{3}(60 \mathrm{mg}, 0.45 \mathrm{mmol})$ in anhydrous $\mathrm{THF}(2 \mathrm{~mL})$ at $0{ }^{\circ} \mathrm{C}$ was added $1,5 \mathrm{~mL}$ solution of $\mathrm{LiAlH}_{4} 1 \mathrm{M}$ in THF. This solution 
was added via cannula to a solution of $\mathbf{8}(100 \mathrm{mg}, 0.28 \mathrm{mmol})$ in THF $(3 \mathrm{~mL})$ at $0{ }^{\circ} \mathrm{C}$. The reaction mixture was quenched with ice-water after $1 \mathrm{~h}$, and extracted with $\mathrm{CHCl}_{3}(20 \mathrm{~mL} \times 3)$, dried over anhydrous $\mathrm{Na}_{2} \mathrm{SO}_{4}$ and concentrated in vacuo to give the crude product: ${ }^{1} \mathrm{H}-\mathrm{NMR}\left(400 \mathrm{MHz}, \mathrm{CDCl}_{3}\right) \delta 6.87(1 \mathrm{H}, \mathrm{s})$, $6.55(1 \mathrm{H}, \mathrm{s}), 5.83(1 \mathrm{H}, \mathrm{d}, J=12.0 \mathrm{~Hz}), 5.69(1 \mathrm{H}, \mathrm{d}, J=12.0 \mathrm{~Hz})$, 4.09-3.95 (4H, m), 3.85 (3H, s, OMe), $3.84(3 \mathrm{H}, \mathrm{s}, \mathrm{OMe}), 3.18-$ $3.11(3 \mathrm{H}, \mathrm{m}), 3.01-2.90(2 \mathrm{H}, \mathrm{m}), 2.62(1 \mathrm{H}, \mathrm{m}), 2.48(1 \mathrm{H}, \mathrm{m})$, $2.12(1 \mathrm{H}, \mathrm{dd}, J=5.2,13.6 \mathrm{~Hz}), 2.04(1 \mathrm{H}, \mathrm{dd}, J=8.4,13.6 \mathrm{~Hz})$, $1.95(1 \mathrm{H}, \mathrm{m}), 1.81(1 \mathrm{H}, \mathrm{m})$. The crude product was dissolved in acetone $(5 \mathrm{~mL})$ followed by addition of $10 \mathrm{mg}$ of TsOH. The reaction solution was heated at $80{ }^{\circ} \mathrm{C}$ for $2 \mathrm{~h}$, and then concent rated under reduced pressure. The resulting residue was purified by silica gel column chromatography $\left(\mathrm{CHCl}_{3} / \mathrm{MeOH} 20: 1\right.$ to $10: 1)$ to yield $9(67 \mathrm{mg}, 80 \%)$ as a colorless liquid. ${ }^{1} \mathrm{H}-\mathrm{NMR}$ $\left(400 \mathrm{MHz}, \mathrm{CDCl}_{3}\right) \delta 6.69(1 \mathrm{H}, \mathrm{s}), 6.68(1 \mathrm{H}, \mathrm{s}), 6.58(1 \mathrm{H}, \mathrm{dd}$, $J=2.0,10.4 \mathrm{~Hz}), 6.04(1 \mathrm{H}, \mathrm{d}, J=10.4 \mathrm{~Hz}), 3.88(3 \mathrm{H}, \mathrm{s}, \mathrm{OMe})$, $3.80(3 \mathrm{H}, \mathrm{s}, \mathrm{OMe}), 3.17-2.84(6 \mathrm{H}, \mathrm{m}), 2.66-2.52(2 \mathrm{H}, \mathrm{m}), 2.07$ $(1 \mathrm{H}, \mathrm{m}), 1.74-1.63(2 \mathrm{H}, \mathrm{m}) .{ }^{13} \mathrm{C}-\mathrm{NMR}\left(100 \mathrm{MHz}, \mathrm{CDCl}_{3}\right) \delta$ $198.6,151.9,147.9,147.6,129.1,127.5,125$.

Acknowledgments. This work was supported by grant (R012007-000-20037-0) from the Basic Research Program of Korea Science and Engineering Foundation, and we appreciate Center for Research for Research Facilities, CNU for the permission to NMR.

\section{References and Footnotes}

1. (a) Tanaka, H.; Tanaka, T.; Etoh, H.; Goto, S.; Terada, Y. Heterocycles 1999, 51, 2759-2764. (b) Dyke, S. F.; Quessy, S. N. The Alkaloids; Rodrigo, R. G. A., Ed.; Academic Press: New York, 1981; Vol. 18

2. (a) Miranda, L. D.; Zard, S. Z. Org. Lett. 2002, 4, 1135-1138. (b) Chikaoka, S.; Toyao, A.; Ogasawara, M.; Tamura, O.; Ishibashi H. J. Org. Chem. 2003, 68, 312-318 and references therein.

3. (a) Allin, S. M.; Streetley, G. B.; Slater, M.; James, S. L.; Martin, W. P. Tetrahedron Lett. 2004, 45, 5493-5496. (b) Allin, S. M.; James, S. L.; Elsegood, M. R. J.; Martin, W. P. J. Org. Chem. 2002, 67, 9464-9467. (c) Padwa, A.; Lee, H. I.; Rashatasakhon, P.; Rose, M. J. Org. Chem. 2004, 69, 8209-8218 and references therein. (d) Katritzky, R. A.; He, H.-Y.; Jiang, R. Tetrahedron Lett. 2002, 43, 2831-2833.

4. (a) Rigby, J. H.; Hughes, R. C.; Heeg, M. J. J. Am. Chem. Soc. 1995, 117, 7834-7835. (b) Rigby, J. H.; Deur, C.; Heeg, M. J. Tetrahedron Lett. 1999, 40, 6887-6890.

5. Dréau, M.-A.; Desmaële, D.; Dumas, F.; dAAngelo, J. J. Org. Chem. 1993, 58, 2933-2935.

6. Kim, G.; Kim, J. H.; Lee K. Y. J. Org. Chem. 2006, 7, 2185-2187.

7. (a) Lee, H. I.; Cassidy, M. P.; Rashatasakhon, P.; Padwa, A. Org. Lett. 2003, 5, 5067-5070. (b) Padwa, A.; Lee, H. I.; Rashatasakhon, P.; Rose, M. J. Org. Chem. 2004, 69, 8209-8218. (c) Shuanhu, G.; Tu, Q. Y.; Hu, X.; Wang, S.; Uua, R.; Jiang, Y.; Zhao, Y.; Fan, X.; Zhang, S. Org. Lett. 2006, 8, 2373-2376.

8. Tsuda, Y.; Nakai, A.; Ito, K.; Suzuki, F.; Haruna, M. Heterocycles 1984, 22, 1817-1820. 